Applied Physics B manuscript No.

(will be inserted by the editor)

\title{
Josephson dynamics for coupled polariton modes under the atom-field interaction in the cavity
}

\author{
A.P. Alodjants ${ }^{1}$, S.M. Arakelian ${ }^{1}$, S.N. Bagayev ${ }^{2}$, V.S. Egorov ${ }^{3}$, A.Yu. Leksin ${ }^{1}$ \\ 1 Vladimir State University, e-mail: alodjants@vpti.vladimir.ru \\ 2 Institute of Laser Physics, Pr.Lavrentyeva 13/3, Novosibirsk, 630090 Russia Fax (3833)33-20-67 \\ 3 St-Petersburg State University
}

'Received: date / Revised version: date

\begin{abstract}
We consider a new approach to the problem of Bose-Einstein condensation (BEC) of polaritons for atom-field interaction under the strong coupling regime in the cavity. We investigate the dynamics of two macroscopically populated polariton modes corresponding to the upper and lower branch energy states coupled via Kerr-like nonlinearity of atomic medium. We found out the dispersion relations for new type of collective excitations in the system under consideration. Various temporal regimes like linear (nonlinear) Josephson transition and/or Rabi oscillations, macroscopic quantum selftrapping (MQST) dynamics for population imbalance of polariton modes are predicted. We also examine the switching properties for time-averaged population imbalance depending on initial conditions, effective nonlinear parameter of atomic medium and kinetic energy of lowbranch polaritons.
\end{abstract}

PACS 03.75.Lm; 71.36.+c, 42.50.Fx;

\section{Introduction}

Novel experiments on observation of Bose-Einstein condensation (BEC) gives new opportunities to investigate of collective coherent phenomena in quantum and atomic optics and solid state physics - see e.g. [1, 2]. An important tool for that can be taken with the help of the Josephson junction problem approach that has a long history relating to Cooper-pair tunneling in the superconductor Josephson junction (SJJ) systems - see e.g. 3]. Various aspects of Josephson phenomenon and superfluid behavior for two weakly linked atomic condensates in double-well potential have been discussed in a number of papers 4 - 7 . However just recently the coherent oscillations and self-trapping regime have been observed experimentally in [8] for two condensates of ${ }^{87} \mathrm{Rb}$ atoms placed in a special optical dipole trap. The principle item is that photonic "condensate" exhibits a similar nonlinear behavior in the problem of optical Joseph- son junction (OJJ) consisting of two tunnel-coupled optical fibers and/or semiconductor waveguides (quantum wells) 9]. Another type of Josephson coupling (nonlinear Josephson effect) for condensate modes has been considered in 10. In particular, nonlinear dynamics like macroscopic self-trapping and intermodal population exchange, has been theoretically analyzed for condensate collective modes (eigenmodes), and namely, for ground state and first excited state modes. The effective coupling of these modes is guarantied by atom-atom scattering, i.e. by the own nonlinearity of BEC placed in highly anisotropic trap.

However, the observation of mentioned above phenomena with pure atomic BEC medium is strictly limited by extremely low temperature (about hundred $\mathrm{nK}$ ) conditions. At present the problem of high-temperature quasi-condensation phenomenon that occurs for cavity excitons and polaritons in so-called strong coupling regime evokes a great interest [1, 11]-14. Speaking more precisely the low branch cavity polaritons can be considered as a weakly interacting two-dimensional Bose-gas for which the Kosterlitz-Thouless phase transition occurs at high enough temperatures due to the extremely small mass of polaritons $m_{\text {eff }}=5 \cdot 10^{-33} \mathrm{~g}$ under condition of strong coupling regime for excitons and quantized e.m. field. Although the polariton condensate is the non-equilibrium state and occurs in a specially control situation, the macroscopic occupation of ground state with momentum $\mathbf{k}=0$ has been examined experimentally for quantum wells inserted in a semiconductor $(\mathrm{CdTe} / \mathrm{CdMgTe})$ microcavity - see [12] and more recent results in [13. The coherence properties have been obtained in [11] for low-branch polaritons above the threshold of generation in semiconductor (Ga-As) microcavity. It has been demonstrated that statistical properties of the excitons can be converted to match the excitons in coherent states.

The problem is that the BEC state in semiconductor systems, fabricated at present, is essentially limited by disorder factor. Recently it was suggested to use a 
A.P. Alodjants et al.

strong disorder in the microcavity to obtain polariton quasi-condensation [15. However it is not clear until now it is possible to achieve true condensation for excitonpolariton substance in semiconductor microcavity for this case.

In our paper [16] it has been shown that polariton true condensation can be achieved for relatively hot atomic vapor in the case of polariton trapping, when atoms are strongly coupled with optical field in the resonator. The degeneration of weakly interacting 2D-gas of polaritons can be also associated with the effect of socalled "spectrum condensation" observed previously for sodium atoms in the cavity.

For some practical purposes the coherent properties of upper branch of polaritons can be important as well. In fact, the problem of formation of quantum entangled polariton states from different branches [17] is the case in respect to construct the algorithms of quantum cloning and storage for optical field into the cavity polaritons being recently proposed [18. However, in the experiment [14] the upper polariton branch has not been developed in the photoluminescence when the quasi-condensation of lower polariton branch takes place under the thermal equilibrium condition. The existence of large energy gap (about ten of meV) between upper and lower branches is also a reason for that.

But the macroscopic population of the ground (low branch) polaritonic state in atomic system can be only one of possible way to obtain interbranch dynamical regimes for such a polariton system. In particular, the energy gap in atomic system can be in $10^{4}-10^{5}$ times smaller then for semiconductor one [16], [19], and some (small) population of upper branch does exist even under the quasi-equilibrium conditions. Upper polaritonic branch can be populated also by using Landau-Zener effect when the tunnelling phenomenon between two eigenstates of the system takes place for the time-dependent detuning - e.g. [21].

Another possibility to observe such a dynamics is determined by a special scheme of coherent excitation of polaritons from different branches 22. Quantum beats and Rabi oscillations in exciton-photon system have been observed in this case 23.

In present paper we consider the problem of nonlinear dynamics for polaritons of different branches under the mean field approximation. Our approach has some analogy with the problem of macroscopic quantum tunneling and interference that takes place for atomic condensate being associated with ground state and first excited state. Actually, low and upper branches of polaritons represent two types of normal modes in atom-field interaction. They can be coupled to each other due to atomic nonlinearity.

The paper is arranged as follows. In Section 2 we establish nonlinear model of interaction of two-level atoms with quantized e.m. field in the cavity for both momentum and coordinate representation - cf. [1], [19], 24].
The adiabatic approximation is discussed for the last wcase. In Section 3 we propose a new approach to describe a nonlinear Josephson junction problem for both upper and lower macroscopic polariton modes coupled in momentum space due to nonlinear (Kerr-like) atomfield interaction. The analysis of Josephson dynamics and switching of various regimes of polariton interaction is presented in Section 4. In Conclusion we briefly summarize our results.

\section{The nonlinear model of atom-field interaction in the cavity}

To describe the interaction of two-level atomic system with a quantum e.m. field, we consider a localized exciton model within the framework of the Dicke-like Hamiltonian for the interaction of the $N$ two-level atoms with a quantized e.m. field (cf. [1, [2], [19]):

$$
\begin{aligned}
H=\sum_{\mathbf{k}} & E_{\mathrm{ph}}(k) \psi_{\mathbf{k}}^{\dagger} \psi_{\mathbf{k}}+\sum_{j}^{N} \frac{E_{\mathrm{at}}}{2}\left(b_{j}^{\dagger} b_{j}-a_{j}^{\dagger} a_{j}\right) \\
& +\sum_{\mathbf{k}} \sum_{j}^{N} \frac{g}{\sqrt{N}}\left(\psi_{\mathbf{k}}^{\dagger} a_{j}^{\dagger} b_{j}+b_{j}^{\dagger} a_{j} \psi_{\mathbf{k}}\right) \\
& +\sum_{\mathbf{k}} \sum_{j}^{N} \frac{\kappa}{\sqrt{N}} \psi_{\mathbf{k}}^{\dagger}\left(\psi_{\mathbf{k}}^{\dagger} a_{j}^{\dagger} b_{j}+b_{j}^{\dagger} a_{j} \psi_{\mathbf{k}}\right) \psi_{\mathbf{k}},
\end{aligned}
$$

where $\psi_{\mathbf{k}}\left(\psi_{\mathbf{k}}^{\dagger}\right)$ is the annihilation (creation) operator for photon with momentum k; $a\left(a^{\dagger}\right)$ and $b\left(b^{\dagger}\right)$ are the annihilation (creation) operators for atoms at the lower $|a\rangle$ and upper $|b\rangle$ levels, respectively, $N$ is the total number of particles, $E_{\text {at }}$ is the energy of atomic transition between the $|a\rangle$ and $|b\rangle$ levels (here we neglect the motion of atoms in the cavity), $E_{\mathrm{ph}}(k)$ defines the dispersion relation for photons in the cavity, and $g$ characterizes the atom-field coupling. The last term with coefficient $\kappa$ in the Hamiltonian (11) takes into account a nonlinear atom-field interaction, and can be considered in the framework of nonlinear Jeans-Cummings model. The interaction implies a polariton-polariton scattering from different branches, and is determined by Kerr-like (optical) nonlinearity of atomic system.

The character of interbranch polariton interaction is determined by sign of effective parameter $g \cdot \kappa N / E_{\text {at }}$. In particular, repulsive particle interaction (with positive scattering length) is realized when $g \cdot \kappa N<0\left(E_{\text {at }}>0\right)$. On the other hand, attractive interaction (with negative scattering length) occurs for $g \cdot \kappa N>0$. In the paper we take into account the positive coupling parameter $g$ only.

In contrast with semiconductor systems we neglect in Hamiltonian (1) by terms describing the nonlinear processes of exciton-exciton scattering - see e.g. [1. Such processes can be reduced to parametric amplification of polaritons and/or stimulated emission - see, e.g. [24]. Our approach is obviously valid for ensemble of non(or weakly) interacting atomic gas for which the average 
interatomic separation length is much larger than characteristic length of interaction between the atoms [25].

For high-reflection mirrors in the cavity the normal (to the plane of the mirrors) component of the photon wave vector $k_{\perp}$ is quantized, i.e. $k_{\perp}=\pi m / L_{\text {cav }}$, where $L_{\text {cav }}$ is the cavity length, $m$ is the number of modes. For the mode component in parallel to the mirror plane $k_{\|}$we have a continuum. In paraxial approximation $\left(k_{\|} \ll k_{\perp}\right)$ the dispersion relation for photon energy $E_{\mathrm{ph}}(k)$ inside the cavity can be represented as follows:

$$
E_{\mathrm{ph}}(k)=\hbar c|\mathbf{k}|=\hbar c \sqrt{k_{\perp}^{2}+k_{\|}^{2}} \approx \hbar c k_{\perp}+\frac{\hbar^{2} k_{\|}^{2}}{2 m_{\mathrm{ph}}},
$$

where $m_{\mathrm{ph}}=\hbar k_{\perp} / c$ is the effective photon mass in the cavity.

In the paper we impose a strong coupling regime for which the cavity modes are coherently coupled with collective atomic exitations that can be introduced with the help of annihilation $(\phi)$ and creation $\left(\phi^{\dagger}\right)$ operators as (cf. [18]):

$$
\phi=\sum_{i=1}^{N} \frac{a_{j}^{\dagger} b_{j}}{\sqrt{N}}, \quad \phi^{\dagger}=\sum_{i=1}^{N} \frac{b_{j}^{\dagger} a_{j}}{\sqrt{N}} .
$$

The low (or zero) density limit which is used for excitons in semi-conductor structures (see e.g. [1]) corresponds to two-level atomic system without inversion. In this case the atomic excitation operators $\phi$ and $\phi^{\dagger}$ in Eqs. (3) obey to usual commutation relation $\left[\phi, \phi^{\dagger}\right] \simeq 1$ for Bose system when the atoms occupy mainly a ground state $|a\rangle$ of energy.

Let us consider the system (11)-(3) of atom-field interaction in the cavity in coordinate representation. In this case we can introduce appropriate Lagrangian density $L$ in the form:

$$
\begin{aligned}
L= & L_{\mathrm{f}}+L_{\mathrm{at}}+L_{\mathrm{int}}, \\
L_{\mathrm{f}}= & \frac{i \hbar}{2}\left(\Psi \frac{\partial \Psi^{*}}{\partial t}-\Psi^{*} \frac{\partial \Psi}{\partial t}\right) \\
& +\frac{i \hbar c}{2}\left(\Psi \frac{\partial \Psi^{*}}{\partial z}-\Psi^{*} \frac{\partial \Psi}{\partial z}\right) \\
& +\frac{\hbar^{2}}{2 m_{\mathrm{ph}}}\left|\nabla_{\perp} \Psi\right|^{2}+U_{\mathrm{opt}}|\Psi|^{2}, \\
L_{\mathrm{at}} & =\frac{i \hbar}{2}\left(\Phi \frac{\partial \Phi^{*}}{\partial t}-\Phi^{*} \frac{\partial \Phi}{\partial t}\right)+E_{\mathrm{at}}|\Phi|^{2}, \\
L_{\mathrm{int}} & =g\left(\Psi^{*} \Phi+\Psi \Phi^{*}\right)+\kappa\left(\Psi^{*} \Phi+\Psi \Phi^{*}\right)|\Psi|^{2},
\end{aligned}
$$

where the $\Psi \equiv \Psi(\mathbf{r}, t)$ function (i.e. the order parameter) describes both the time evolution and spatial distribution of optical field in the cavity. In paraxial approximation (2) under the mean field theory condition the value of $\Phi \equiv \Phi(t)$ is the $\mathrm{C}$-number that characterizes a macroscopic excitation in time of atomic system (here we neglect the motion and space distribution of atomic cloud in the cavity). In expression (4) we also introduce the external (harmonic) potential $U_{\text {opt }} \equiv U_{\text {opt }}(x, y)$ for the photon trapping - cf. [16].

Using the expression (44) under the variational approach (see e.g. [9]) we obtain the following coupled equations for the field $\Psi$ and the atomic excitation $\Phi$ respectively:

$$
\begin{aligned}
& i \hbar\left(\frac{\partial}{\partial t}+c \frac{\partial}{\partial z}\right) \Psi= \\
& \quad=\left(-\frac{\hbar^{2}}{2 m_{p h}}\left(\frac{\partial^{2}}{\partial x^{2}}+\frac{\partial^{2}}{\partial y^{2}}\right)+U_{o p t}(x, y)\right) \Psi \\
& \quad+g \Phi+\kappa\left(2|\Psi|^{2} \Phi+\Phi^{*} \Psi^{2}\right)
\end{aligned}
$$

$$
i \hbar \frac{d \Phi}{d t}=E_{a t} \Phi+g \Psi+\kappa|\Psi|^{2} \Psi
$$

Let us consider a solution of Eqs. (5), (6) under the adiabatic approximation for which we suppose $d \Phi / d t=0$ for atomic excitation. Eliminating the $\Phi$ variable from Eq. (6) we arrive to self-consistent generalized Schrödinger equation for the order parameter $\Psi$ that has the form:

$$
\begin{aligned}
& i \hbar\left(\frac{\partial}{\partial t}+c \frac{\partial}{\partial z}\right) \Psi=\left[-\frac{\hbar^{2}}{2 m_{\mathrm{ph}}}\left(\frac{\partial^{2}}{\partial x^{2}}+\frac{\partial^{2}}{\partial y^{2}}\right)\right. \\
& \left.\quad+U_{\mathrm{opt}}(x, y)+u|\Psi|^{2}+d|\Psi|^{4}\right] \Psi
\end{aligned}
$$

where $u=-4 g \kappa / E_{\text {at }}, d=-3 \kappa^{2} / E_{\text {at }}$. Here we also omit the term with $g^{2} / E_{\text {at }}$ that can be also eliminated with help of simple unitary transformation.

Equation (7) describes a behavior of optical beam interacting with nonlinear atomic medium in the cavity. The last term in Eq. (7) can be discarded within the limit $|d||\Psi|^{2} \ll|u|$ that corresponds to phenomenological (macroscopic) approach for weakly interacting Bose-gas of photons in the cavity - cf. [26]. The coefficient $u$ that is determined by Kerr-like nonlinearity of atomic vapor is a control parameter for that.

The coefficient $d$ that is always negative in Eq. (7) characterizes a quintic nonlinearity of the medium. Recently a superfluid behavior has been considered for such a system in 27. In particular, formation of stable vortex lattices for "photonic condensate" has been numerically proposed even for the case without the external trapping potential $U_{\text {opt }}(x, y)$ use.

In general case when both relaxation rate of optical field $\Gamma_{\mathrm{f}}$ and atomic excitations dephasing rate $\Gamma_{\text {ex }}$ are under consideration the adiabatic approach is valid when an inequality

$$
\Gamma_{\mathrm{ex}} \gg \Gamma_{\mathrm{f}}
$$

is hold - cf. [28].

In the present paper we consider the atom-field interaction in the limit when the rates $\Gamma_{\mathrm{ex}}$ and $\Gamma_{\mathrm{f}}$ are the same order of magnitude. In this case we can examine the system described by Hamiltonian (1) using in fact the two macroscopically populated polaritonic modes from different brunches. 


\section{Boson Josephson junction (BJJ) model for polaritons in the cavity}

Let us consider a Josephson dynamics for two modes, i.e. upper and low branch polaritons, those are coherently excited in the cavity - cf. 23]. We suppose that one quantum optical mode (with $\mathbf{k}=0$ as a limit) is only macroscopically occupied. Thus, in Hamiltonian (1) we switch over to macroscopic (continuous) variable $\psi(\mathbf{k})$ for optical field describing this mode. In this case we introduce the time-dependent classical wave functions $\Phi_{1,2}(t) \equiv \sqrt{N_{1,2}(t)} e^{i \theta_{1,2}(t)}$ as:

$$
\begin{aligned}
& \Phi_{1}=\frac{1}{\sqrt{2}}(\psi(\mathbf{k})-\phi), \\
& \Phi_{2}=\frac{1}{\sqrt{2}}(\psi(\mathbf{k})+\phi),
\end{aligned}
$$

that characterizes the bosonic quasi-particles in atomic medium corresponding to upper and lower branch polaritons; $N_{1,2}(t)$ is the time dependent average number of polaritons for upper $\left(N_{1}\right)$ and lower $\left(N_{2}\right)$ branches respectively, $\theta_{1}$ and $\theta_{2}$ are the phases.

Now we introduce the new variables $\varsigma$ and $\theta$ as

$$
\varsigma=\frac{N_{1}-N_{2}}{N_{\mathrm{ex}}}, \quad \theta=\theta_{2}-\theta_{1}
$$

that characterize a fractional state population imbalance $(\varsigma)$ and relative phase $(\theta)$ for polaritonic modes respectively, $N_{\text {ex }}=N_{1}+N_{2}$ is the total number of polaritons in the cavity.

In the paper we pay our attention to the problem of polariton interaction along the cavity length under the atom-field resonance condition $\Delta \equiv E_{\text {at }}-\hbar c\left|k_{\perp}\right|=0$. The Hamiltonian (1), describing the superfluid dynamics of the polaritons in momentum representation, can be evaluated in the terms of new variables $\varsigma$ and $\theta$ as

$$
\begin{aligned}
H= & \Lambda_{\mathrm{eff}} \beta \varsigma^{2}-\Lambda_{\mathrm{eff}} \beta\left(\sqrt{1-\varsigma^{2}} \cos \theta-\sqrt{1+\beta^{2}}\right) \\
& \times \sqrt{1-\varsigma^{2}} \cos \theta+\Delta E_{\mathrm{eff}},
\end{aligned}
$$

where $\beta=E_{\mathrm{tr}} /|g|$ is the scaled (normalized) energy of polaritons in transverse plane, $E_{\mathrm{tr}}=\hbar^{2} k_{\|}^{2} /\left(2 m_{\mathrm{pol}}\right)$ is their kinetic energy producing the effect of quantum pressure, $m_{\mathrm{pol}} \simeq 2 m_{\mathrm{ph}}$ is the mass of polaritons in the cavity. It is possible to define Josephson-like length $\lambda_{\mathrm{J}}=$ $\sqrt{|\beta|} / k_{\|}$that specifies a spatial control parameter in the system - cf. [3], 5]. For strong coupling regime with the value of atom-field coupling parameter $g \simeq 0.05 \mathrm{meV}$ the length $\lambda_{\mathrm{J}} \simeq 11 \mu \mathrm{m}$. The result is the same as for atomic BEC in the BJJ problem - cf. [5]. The crucial dimensionless parameter $\Lambda_{\text {eff }}=\kappa N_{\text {ex }} /\left[2|g|\left(1+\beta^{2}\right)\right] \equiv$ $\Lambda /\left(1+\beta^{2}\right)$ determines a nonlinear Josephson coupling between polaritons of different branches (the parameter $\Lambda<0$, i.e. $\kappa N_{\mathrm{ex}}<0$, for positive scattering length of polaritons).
In expression (12) for Hamiltonian $H$ we define an effective zero-point energy difference $\Delta E_{\text {eff }}$ as

$$
\Delta E_{\mathrm{eff}}=\alpha-\Lambda_{\mathrm{eff}} \sqrt{1-\varsigma^{2}}\left(1-\beta^{2}\right) \cos \theta,
$$

where we have made a denotation $\alpha=\sqrt{1+\beta^{2}}\left(1+\Lambda_{\mathrm{eff}}\right)$. Physically, the parameter $\alpha$ characterizes the energy gap between polariton branches, i.e. determines the unalterable zero point energy difference when condition $\beta=1$ takes place.

Using the Hamiltonian (11) and definition (13) for $\Delta E_{\text {eff }}$ one can obtain the following equations for canonically conjugated variables $\varsigma$ and $\theta$ :

$$
\begin{aligned}
\frac{d \varsigma}{d \tau}= & -\Lambda_{\mathrm{eff}}\left(\varsigma\left(1-\beta^{2}\right)-\beta \sqrt{1+\beta^{2}}\right. \\
& \left.+2 \beta \sqrt{1-\varsigma^{2}} \cos \theta\right) \sqrt{1-\varsigma^{2}} \sin \theta, \\
\frac{d \theta}{d \tau}= & \alpha-\Lambda_{\mathrm{eff}}\left(\frac{1-2 \varsigma^{2}}{\sqrt{1-\varsigma^{2}}}\right) \cos \theta \\
+ & 2 \Lambda_{\mathrm{eff}} \beta\left(\varsigma-\frac{\sqrt{1+\beta^{2}} \varsigma}{2 \sqrt{1-\varsigma^{2}}} \cos \theta+\varsigma \cos ^{2} \theta\right. \\
+ & \left.\frac{\beta}{2}\left(\frac{1-2 \varsigma^{2}}{\sqrt{1-\varsigma^{2}}}\right) \cos \theta\right),
\end{aligned}
$$

where $\tau=g t / \hbar$ is the dimensionless time coordinate.

Thus, the set of equations (14), (15) represents an extension of the BJJ model in nonlinear dynamics approach with the phenomenon of tunneling for polaritons from different branches. In the case $\beta=0$ the Eqs. (14), (15) describe the polariton arising effect along the cavity length only.

Obviously, it is possible to establish some link between the system of Eqs. (14), (15) describing the polariton dynamics in the cavity and the other Josephson dynamical systems - cf. [3] - [10]. In particular, the equation (14) characterizes the interbranch current $I$ for polaritons. In general it has a complex form. However we can represent the current $I$ for small values of $\beta$ as

$$
\begin{gathered}
I \simeq-I_{\mathrm{c}} \sqrt{1-\varsigma^{2}} \sin \theta+J_{1} \varsigma \sqrt{1-\varsigma^{2}} \sin \theta \\
+I_{\mathrm{c}}\left(1-\varsigma^{2}\right) \sin 2 \theta,
\end{gathered}
$$

where $I_{\mathrm{c}}=|\kappa| N_{\mathrm{ex}} E_{\mathrm{tr}} /(2|g| \hbar)$ is the positive critical current for polaritons in transverse plane (we took $\Lambda_{\text {eff }}<$ $0), J_{1}=|\kappa| N_{\mathrm{ex}} /(2 \hbar)$ is the current characterizing the polaritons along the cavity length.

In general the interbranch current $I$ is essentially nonlinear one; the case is quite different from the Cooperpair current in the SJJ problem - cf. 3, 4. From Eq. (16) it is easy to see that the first term, describing the supercurrent of polaritons in transverse plane, is completely similar to the BJJ inertrap current occurring for atomic condensates. The last term in Eq. (16) represents a nondissipative current determined by interaction of the polaritons inside the cavity. Physically, this term can be associated with the second order contribution to Josephson current due to the effect of tunneling for atoms in condensate state to noncondensate quasiparticles in the 
BJJ problem [7]. For small population imbalance $|\varsigma| \ll 1$ the contribution to the current of polaritons along the cavity length can be neglected .

Energy difference $\Delta E_{\text {eff }}$ defined in Eq. (13) plays a principal role in total polariton dynamics. In respect of the atomic BJJ problem the zero-point energy difference $\Delta E_{\text {eff }}$ is determined by both the geometry of the trap and the nonlinearity of the atomic condensates 5 , 6]. The energy $\Delta E_{\text {eff }}$, determined by applied voltage across the junctions, results in oscillations being in analogy with the ac-Josephson frequency $\omega_{\mathrm{ac}} \sim \Delta E_{\text {eff }}$ in the SJJ problem. However in the case of polaritons, the energy difference $\Delta E_{\text {eff }}$ in Eq. (13) is mostly depends on dynamical variables $\varsigma$ and $\theta$ in general. Physically, such a behavior is caused by nonlinear (Kerr-like) crossinteraction of polaritons from different branches in the cavity. The energy difference $\Delta E_{\text {eff }}$ defined in Eq. (13) can be suppressed for the case $\Lambda_{\text {eff }}<0$. For example, we have $\Delta E_{\text {eff }}=0$ for the values $\beta=1, \Lambda_{\text {eff }}=-1$ what implies the polaritons with momentum $k_{\|} \simeq 1 / \lambda_{\mathrm{J}}$ and kinetic energy $E_{\mathrm{tr}}=|\kappa| N_{\mathrm{ex}} / 4$. This condition is compatible with the requirement for definition of the healing (or coherent) length $\xi_{\mathrm{c}}$ that plays an important role for superfluid systems - cf. 25. In particular, a typical length scale $\xi_{\mathrm{c}}$ for that is determined by the condition when the $E_{\text {tr }}$ energy is of the order of the interaction energy for condensate atoms (determined by the $\kappa N_{\text {ex }}$ parameter). For the case under discussion we have the coherent length $\xi_{\mathrm{c}} \sim \lambda_{\mathrm{J}}$.

\section{Nonlinear superfluid dynamics for trapped polaritons}

\subsection{Small-amplitude oscillations}

We start our analysis from the case of non-interacting (ideal) gas of polaritons when $\Lambda=0$. The solutions of Eqs.(14), (15) are simply presented in the form:

$$
\theta=g \sqrt{1+\beta^{2}} t \equiv \frac{\Delta E}{2} t, \quad \varsigma=\varsigma(0),
$$

where $\Delta E=2 g \sqrt{1+\beta^{2}}$ is the energy splitting for noninteracting polaritons, $\varsigma(0)$ is the initial population difference. Thus, in the case under consideration the polariton branches are not overlapped and therefore the polaritons arising in the problem are still uncoupled.

Now let us consider a small-amplitude oscillations case for population imbalance $\varsigma$ when the time average values $\langle\theta\rangle=0$ ("zero phase" oscillations) or $\langle\theta\rangle=\pi$ (" $\pi$-phase" oscillations). Linearizing the Eqs. (14), (15) in respect of the $\varsigma$ and $\theta$ variables we arrive to equation

$$
\frac{d^{2} \varsigma}{d \tau^{2}}+\Omega_{0, \pi}^{2} \varsigma=F_{0, \pi}
$$

where two parameters, i.e. dimensionless angular frequencies of oscillations $\Omega_{0, \pi}$ and external forces $F_{0, \pi}$, are defined as

$$
\begin{gathered}
\Omega_{0, \pi}=\left(\Omega_{\mathrm{JP} 0, \pi}^{2} \pm \Omega_{\mathrm{R} 0, \pi}^{2}\right)^{1 / 2}, \\
\Omega_{\mathrm{JP} 0, \pi}=\sqrt{E_{\mathrm{J}}^{0, \pi} E_{\mathrm{C}}^{0, \pi}}, \\
\Omega_{\mathrm{R} 0, \pi}=\sqrt{\Lambda_{\mathrm{eff}}\left(1-\beta^{2}\right)\left(\alpha \mp \Lambda_{\mathrm{eff}} \pm \Lambda_{\mathrm{eff}} \beta^{2}\right)}, \\
F_{0, \pi}=-E_{\mathrm{J}}^{(0, \pi)}\left(\alpha \mp \Lambda_{\mathrm{eff}} \pm \Lambda_{\mathrm{eff}} \beta^{2}\right) .
\end{gathered}
$$

In expressions (19)-(22) we introduce two characteristic (dimensionless) parameters, i.e. the Josephson coupling $\operatorname{energy} E_{\mathrm{J}}^{(0, \pi)}=\Lambda_{\text {eff }} \beta\left(2 \mp \sqrt{1+\beta^{2}}\right) \simeq I_{\mathrm{c}}$, and "capacitive" energy $E_{\mathrm{C}}^{(0, \pi)}=\Lambda_{\mathrm{eff}} \beta\left(4 \mp \sqrt{1+\beta^{2}}\right)$ that describe the polariton nonlinear interaction for different branches - cf. 8 .

The expressions (19)-(21) determine the dispersion relations for a new type of collective excitations arising in the cavity for polariton interaction (we stress here the fact that $\beta$-parameter is proportional to momentum as $\left.\beta \sim k_{\|}^{2}\right)$. Nevertheless it is useful to establish some link between the problem considered here and some other Josephson junction systems. In particular, the small amplitude oscillation frequency regime, described by Eq. (19), has the same structure as a frequency of small oscillations for the atomic population imbalance occurring for Bose-Einstein condensate confined in the $\mathrm{W}$-potential $[5]$. The second term in Eq. (19) characterizes the linearized Rabi oscillations with frequency $\Omega_{\mathrm{R} 0, \pi}$ of the population imbalance. The angular frequency $\Omega_{\mathrm{JP}}$ in Eqs. (19), (20) can be considered as an analog of the plasma frequency for Josephson plasmon in the problem of a Cooper pair macroscopic tunneling process across the junction - cf. [3], 29. The driving force $F_{0, \pi}$ depends on $\beta$-parameter that determines quantum pressure of polaritons in transverse spatial plane.

We distinguish two principal regimes of collective amplitude oscillations for the cavity polaritons. Namely, within the limit $\Omega_{\mathrm{R}} \gg \Omega_{\mathrm{JP}}$ so called Rabi regime occurs. For quasiparticles with a small momentum $k_{\|}$(i.e. for $\left.\beta^{4} \ll 1\right)$ the population imbalance oscillates with angular frequency $\Omega_{0} \approx \sqrt{4.5 \Lambda_{\text {eff }}^{2} \beta^{2}-0.5 \Lambda_{\text {eff }} \beta^{2}+\Lambda_{\text {eff }}}$ vs $\tau$ under the zero phase regime. In the case of absence of polaritons in transverse plane $(\beta=0)$ we have $\Omega_{0}=\Omega_{\mathrm{R} 0}=\sqrt{\Lambda}$ (for $\Lambda>0$ ). The expression (20) and numerical simulation show that the Josephson coupling energy $E_{J}$ is vanished, and the contribution of Rabi frequency $\Omega_{\mathrm{R}}$ in Eq. (19) is important for parameter $\beta \rightarrow 1.7$.

On the other hand, the Josephson regime is realized for the polaritons in the cavity within the limit $\Omega_{\mathrm{R}} \ll$ $\Omega_{\mathrm{JP}}$. Such a regime of polariton oscillations is achieved for the parameter $\beta=1$.

In the limit of large $\beta\left(\beta^{2} \gg 1\right)$, i.e. for the quasiparticles with large momentum $k_{\|}$, the frequency of amplitude oscillations is $\Omega_{0} \simeq \sqrt{\Lambda_{\text {eff }}^{2} \beta^{4}+\left|\Lambda_{\text {eff }}\right| \beta^{3}}=$ $\sqrt{\sqrt{\left|\Lambda_{\text {eff }}\right| \beta} E_{\mathrm{C}} \Omega_{\mathrm{R}}+\Omega_{\mathrm{R}}^{2}}$ (for $\Lambda_{\text {eff }}<0$ ) that is in good 
agreement with the oscillation frequency expression obtained for atomic population imbalance in the BJJ problem 29]. Thus, crossover to Rabi oscillations with frequency $\Omega_{\mathrm{R}} \simeq \sqrt{\left|\Lambda_{\mathrm{eff}}\right| \beta^{3}}$ (for $\left|\Lambda_{\mathrm{eff}}\right| \beta \ll 1$ ) can be easily achieved by varying, for example, of the $\Lambda$-parameter (i.e. the intensity of laser field).

The solution of Eq. (18) is given by following expression:

$$
\varsigma_{0, \pi}=C \cos \left(\Omega_{0, \pi} \tau\right)+\varsigma_{\mathrm{dis}}^{(0, \pi)},
$$

where $C$ is the constant determined by initial conditions, $\left\langle\varsigma_{0, \pi}(\tau)\right\rangle=\varsigma_{\text {dis }}^{(0, \pi)}=F_{0, \pi} / \Omega_{0, \pi}^{2}$ characterizes the displacement for the time average fractional population imbalance $\langle\varsigma\rangle$ in respect of the zero value. The displacement $\varsigma_{\text {dis }}^{(0, \pi)}=0$ and the driving forces $F_{j}=0$ for $\beta=0$ or $\beta=1, \Lambda_{\text {eff }}=-1$. In this case small amplitude oscillations of the $\varsigma$ variable occur around the value $\langle\varsigma\rangle=0$.

Developed above approach can be violated, for example, for running-phase regime when, first, the phase difference $\theta$ grows unrestrictedly and, second, the essentially nonlinear oscillations of population imbalance $\varsigma$ take place. We are also emphasis here some difficulties to obtain so-called Fock regime by using the Hamiltonian (1) when fulfillment of the inequality $E_{\mathrm{c}} \gg E_{\mathrm{J}}$ is required - cf. [29].

\subsection{Stationary states of polaritons}

First, we focus our attention on the out-of-phase stationary states of polaritons in the cavity determined by the conditions $\sin \theta \neq 0, \cos \theta \neq 0$. From (14), (15) it is easy to obtain the following solutions

$$
\begin{aligned}
& \varsigma_{0}=-\frac{\beta}{\Lambda} \frac{\Lambda+2}{\sqrt{\beta^{2}+1}}, \\
& \theta_{0}=\arccos \frac{\Lambda-\beta^{2}+1}{\operatorname{sgn}(\Lambda) \sqrt{\Lambda^{2}-4 \beta^{2}(\Lambda+1)}},
\end{aligned}
$$

that represents unstable saddle point with energy $H_{0}=$ $-\frac{\beta}{\Lambda}(\Lambda+1)$. The existence of solutions (24) implies fulfillment of conditions for population imbalance $\varsigma$ and phase $\theta$, i.e. $|\varsigma| \leq 1,|\cos \theta|<1$. The fact results in certain condition for parameters $\beta$ and $\Lambda$ that looks like:

$$
\Lambda<-\frac{1}{2}\left(\beta^{2}+1\right)
$$

Second, we consider another out-of-phase solution of Eqs. (14), (15) with $\sin \theta= \pm 1$. For stationary fractional population imbalance $\varsigma$ and energy $H$ of the system we find:

$$
\begin{gathered}
\varsigma_{1,2}= \pm 1, \\
H_{1,2}= \pm \sqrt{\beta^{2}+1} \pm \frac{\beta^{2}+1}{ \pm 2 \beta+\sqrt{\beta^{2}+1}}\left(1 \pm \frac{\beta}{\sqrt{\beta^{2}+1}}\right), \\
\varsigma_{3}=\frac{\beta \sqrt{\beta^{2}+1}}{1-\beta^{2}}=-\frac{\left(\Lambda+\beta^{2}+1\right) \sqrt{\beta^{2}+1}}{2 \Lambda \beta}, \\
H_{3}=\frac{\beta^{3}}{1-\beta^{2}} .
\end{gathered}
$$

The solutions (26), (27) do exist if the parameters $\beta$ and $\Lambda$ fulfill the conditions :

$$
\Lambda=\frac{\left(\beta^{2}+1\right)^{3 / 2}}{ \pm 2 \beta+\sqrt{\beta^{2}+1}} \text { for } \varsigma_{1,2},
$$

and

$$
\Lambda=\beta^{2}-1 \text { for } \varsigma_{3},
$$

where $\beta \in\left[-\frac{1}{\sqrt{3}} ; \frac{1}{\sqrt{3}}\right]$. Thus, the solutions (26), (27) represent the unstable saddle points in phase portrait.

Third, Eqs. (14), (15) have zero-phase (in-phase) and $\pi$-phase stationary solutions with the phase $\theta$ determined by the condition $\sin \theta=0$. The stationary points in this case can be found out from solution of algebraic equation

$$
A_{1} \varsigma^{4}+A_{2} \varsigma^{3}+A_{3} \varsigma^{2}+A_{4} \varsigma+A_{5}=0
$$

for fractional population imbalance $\varsigma$, where the real coefficients $A_{j} \equiv A_{j}(\Lambda, \beta)$ are the functions of $\Lambda$ and $\beta$ (we are not directly represent them in the paper due to complexity of the relevant expressions). In general, there exist four roots of Eq. (30) of which only the real solutions are of interest. Note, that one of the solutions of (30) may become a saddle point if the condition (25) is not fulfilled.

\subsection{Phase plane portrait description}

From mathematical point of view the equations (14), (15) describe an undamped nonrigid pendulum with phase $\theta$ and canonical momentum $p_{\theta} \equiv \varsigma$. In the paper we consider a phase-space description to understand the dynamics of the polariton interaction in the cavity. In Fig.1 the evolution of the phase portraits for various values of normalized kinetic energy $\beta$ of polaritons is presented for the same nonlinear $\Lambda$-parameter. The trajectories are plotted for different values of initial conditions for the population imbalance and the phase with $\Lambda$ and $\beta$ parameters kept constant. Closed loop trajectories in the gray area marked in Fig.1 correspond to periodic oscillations of pendulum coordinate and phase in terms of nonlinear pendulum analogy. The stationary points $S_{j}$ $(j=1, \ldots, 6)$ correspond to the roots of Eq.(30). White color regions 1 in Fig.1 determine the running phase regimes that correspond to anharmonic rotation of nonrigid pendulum with relative phase $-\infty<\theta<+\infty$. Stationary saddle points $P_{1,2}$ at the separatrixes in Fig.1a,b are determined from expressions (24) respectively.

For arbitrary value of zero-point energy difference $\Delta E_{\text {eff }}$ defined in (13) the phase-plane portraits are asymmetric as a rule. However, the phase portrait established in Fig.1b becomes completely symmetric for $\Delta E_{\text {eff }}=0$. In particular, the small amplitude zero phase Josephson oscillations of fractional population imbalance take place here in the region 5 with the frequency $\Omega_{0}=\Omega_{\mathrm{JP}}$ 


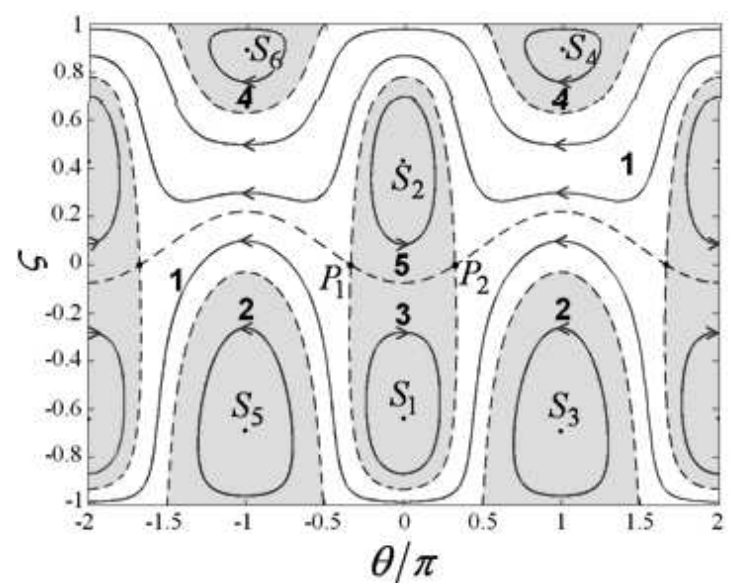

(a)

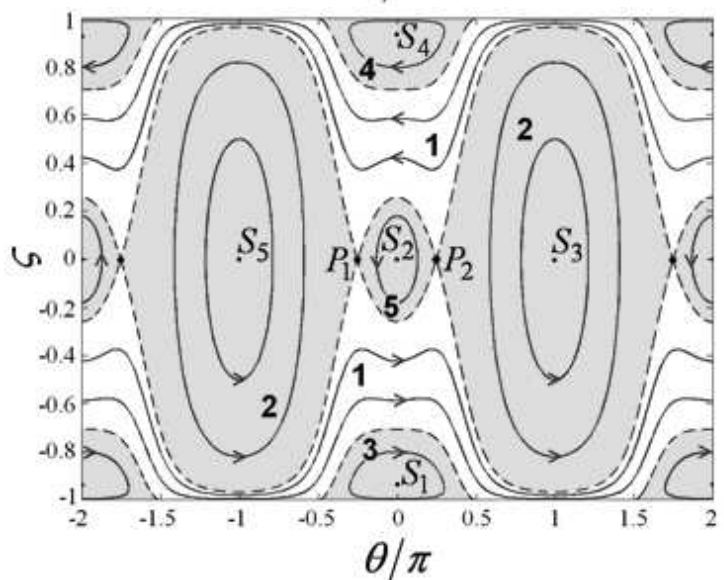

(b)

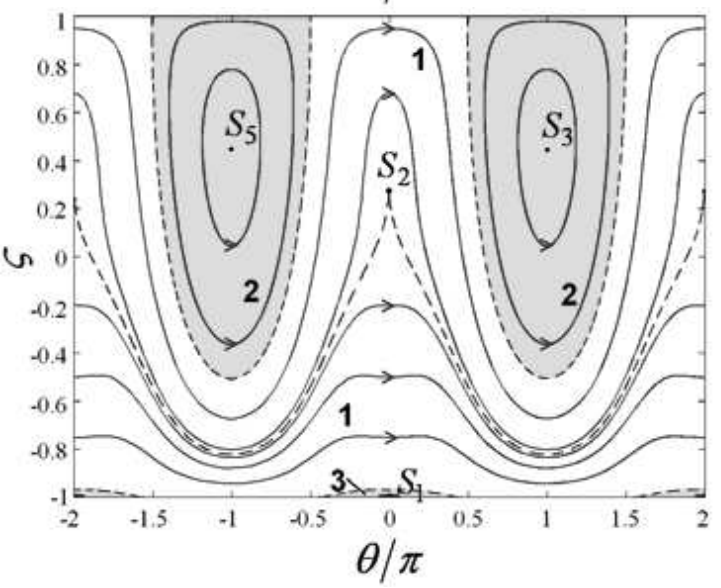

Fig. 1 Phase-plane portraits of the variables $\varsigma$ and $\theta / \pi$ for: a) $\beta=0.15$, b) $\beta=1$, c) $\beta=2.08475$. For all cases the parameter $\Lambda=-2$. The points $S_{j}$ and $P_{j}$ are the stationary points. The regions numerated as ' $1,2, \ldots, 5$ ' determine the various dynamical regimes separated by dashed curves (separatrixes).

around the average value $\langle\varsigma\rangle=0$ with zero displacement. The fact is in agreement with Eqs. (19)-(23). Two symmetric regions numerated as 2 in Fig.1b characterize the large-amplitude ( $\pi$-phase) oscillations of pendulum around the top of vertical axis. A complete energy transfer between polaritons from different branches takes place in this case approximately. For small initial val-

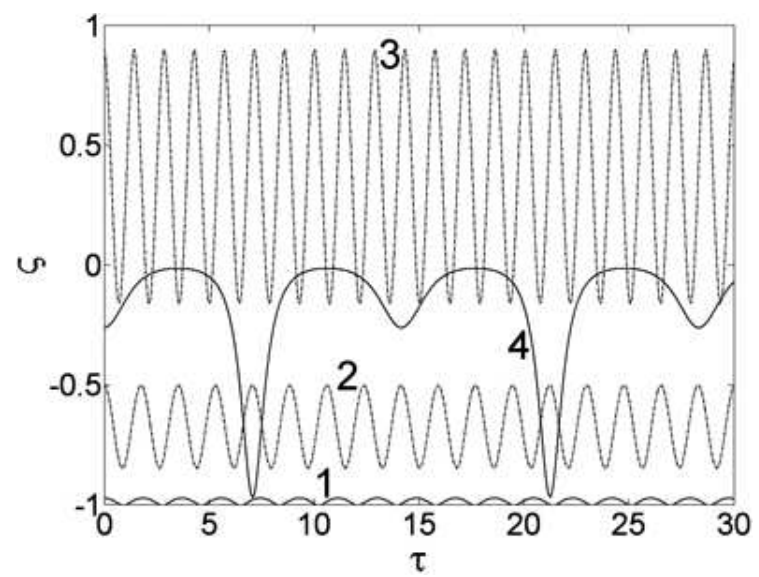

Fig. 2 Fractional population imbalance $\varsigma$ against scaled dimensionless time $\tau$. The parameters are: $\beta=2.08475$, $\varsigma(0)=-0.97, \theta(0)=0$ for curve $1, \beta=0.15, \varsigma(0)=-0.5$, $\theta(0)=\pi$ for curve $2, \beta=2.08475, \varsigma(0)=0.9, \theta(0)=\pi$ for curve 3 and $\beta=1, \varsigma(0)=-0.259, \theta(0)=0$ for curve 4 . For all cases the parameter $\Lambda=-2$.

ues of the fractional population imbalance $\varsigma^{2}(0) \ll 1$ we obtain the small-amplitude $\pi$-phase oscillations with frequency $\Omega_{\pi}$ - see (19).

Other regions in Fig.1b and all of them in Fig.1a,c specify so-called macroscopic quantum self-trapping regimes (MQST) for which a fractional population imbalance $\varsigma$ oscillates around the non-zero value $\langle\varsigma\rangle \neq 0$. It implies that population of either of polariton branches is never completely depleted. In fact, in our case a macroscopic quantum self-trapping behavior of polaritonic modes takes place, first, as a result of nonlinear interaction of macroscopically large number of polaritons from different branches. Second, the MQST regime occurs as a result of the "external force" action (see (18), (23)) depending on effective difference $\Delta E_{\text {eff }} \neq 0$ in (13).

In first case, the dependence on normalized time $\tau$ for fractional population imbalance $\varsigma$ is presented in Fig.2 to visualize the various MQST regimes. In fact, the MQST effect at the phase $\theta=0$ occurs for several cases: in two regions 3,5 in Fig.1a, in regions 3,4 in Fig.1b, and in region 3 in Fig.1c. The curve 1 in Fig. 2 demonstrates a selftrapping behavior of polariton interaction for last case. The regimes discussed here appear for localized (periodically) phase. A similar dynamic MQST behavior occurs for nonlinear interaction of two atomic BEC modes (fundamental and first excited) under the coupled two-mode approximation - cf. [10].

The second kind of the MQST regime associated with so called $\pi$-phase MQST mode is presented in Fig.1 for relative phase values $\theta= \pm \pi$. The gray-color regions marked as 2, 4 in Fig.1a and as 2 in Fig.1c are responsible for that and characterize the $\pi$-phase pendulum rotations with closed loop trajectories around the value $\langle\varsigma\rangle \neq 0$ - cf. [5]. In Fig.2 the curves 2,3 demonstrate the MQST dynamics discussed in respect of the regions taken from the Fig.1a and Fig.1c. The curve 3 describes 
the large amplitude MQST oscillations appearing here for the time-averaged population imbalance $\langle\varsigma\rangle \neq 0$ displacement as a result of presence of zero-point energy difference $\Delta E_{\text {eff }} \neq 0$.

We are emphasis here the two types of the $\pi$-phase modes occurring in the MQST regions in Fig.1a,c for the phase values $\theta= \pm \pi$. Two various dynamical trapping regimes (above and beyond the stationary $\varsigma$-symmetry breaking value of population imbalance $\varsigma_{\mathrm{s}}$ ) have been firstly discussed for macroscopic atomic trapping in the BJJ problem [5]. The modified conditions for that can be represented as follows (cf. [6]):

$$
\langle\varsigma\rangle<\varsigma(0)
$$

for I type of MQST, and

$$
\langle\varsigma\rangle>\varsigma(0)
$$

for II type of MQST. At the stationary (threshold) point $\varsigma_{\mathrm{s}}$ we have $\langle\varsigma\rangle=\varsigma(0)=\varsigma_{\mathrm{s}}$, i.e. the average population imbalance coincides with it initial value $\varsigma(0)$.

In contrast with the BJJ problem in our case for atomic condensate $\pi$-phase modes one can obtain a new type of zero-phase MQST modes when the system demonstrates the self-trapping effect according to the conditions (31), (32). In fact, we speak about the MQST states appearing in the regions 3,5 (Fig.1a), in regions 3,4 (Fig. 1b) and in region 3 (Fig.1c). For stationary points $S_{1,4}$ in Fig.1b we have $\langle\varsigma\rangle=\varsigma_{\mathrm{s}}$.

Finally, third kind of the MQST dynamical regime occurs for various running phase modes with unbounded phase $\theta$. The case is presented by white-color regions 1 in Fig.1. The curve 4 in Fig.2 characterizes the MQST appearing in the region 1 in Fig.1b.

\subsection{Switching dynamics for polaritons}

The MQST regimes under consideration can be achieved by different ways depending on the crucial parameters $\beta, \Lambda$ and also on the values of initial conditions for relative phase $\theta(0)$ and fractional population imbalance $\varsigma(0)$. Practically we can vary one of them keeping others unchanged. For example, let us consider transition from the regime 5 of the Rabi oscillations in Fig.1b upward, i.e. to the MQST regime 1 with unbounded running phase. We can establish the condition for such a transition in this particular case in the form:

$$
H(\varsigma(0), \theta(0))>H_{\text {sep }},
$$

where $H(\varsigma(0), \theta(0))$ is the initial (conserved) energy of the system defined in (12), the energy $H_{\text {sep }}=-\frac{\beta}{\Lambda}(1+\Lambda)$ characterizes the state that corresponds to two separatrixes going through the points $P_{1,2}$ in Fig.1b. For instance, in the case when the parameters $\Lambda, \beta$ and initial phase $\theta(0)$ are unchanged the bound energy $H_{\text {sep }}=0.5$



(a)

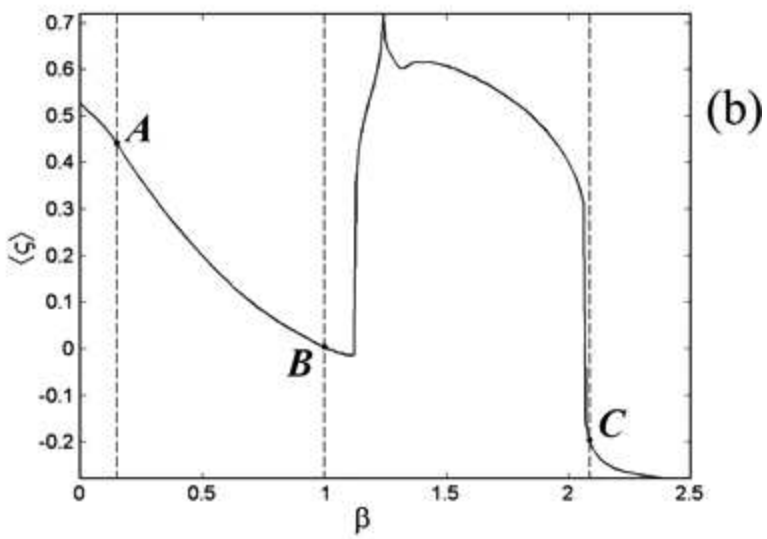

Fig. 3 Time-averaged population imbalance $\langle\varsigma\rangle$ versus (a) $\varsigma(0) / \varsigma_{\mathrm{c}}$ and $(\mathrm{b})-\beta$-parameter. The parameters for $(\mathrm{a})$ are: $\varsigma_{\mathrm{c}} \simeq 0.2588, \beta=1$, and for $(\mathrm{b})-\varsigma(0)=0.2$. In all cases parameter $\Lambda=-2$ and initial phase $\theta(0)=0$.

determines a critical value of fractional relative population imbalance $\varsigma_{\mathrm{c}} \approx 0.2588$. The MQST dynamics occurs in the case when initial value $\varsigma(0)>\varsigma_{c}$.

In Fig.3a we plot the dependence for time-averaged population imbalance $\langle\varsigma\rangle$ as a function on ratio $\varsigma(0) / \varsigma_{\mathrm{c}}$. The value $\langle\varsigma\rangle=0$ in Fig.3a characterizes a polariton interaction for the region 5 in Fig.1b. Sharp changing of population imbalance at $\varsigma(0)=\varsigma_{\mathrm{c}}$ implies the "phase transition" to the MQST state with $\langle\varsigma\rangle \neq 0$ (region 1 ). By similar manner one can obtain a switching effect for dynamical regimes by varying of the $\Lambda$-parameter.

In Fig.3b we establish a switching phenomenon for the time-averaged population imbalance $\langle\varsigma\rangle$ depending on $\beta$-parameter (i.e. on kinetic energy of polaritons in transverse plane). The points A,B,C mark the values of $\beta$-parameter for the phase portraits presented in Fig.1. Initial value of $\langle\varsigma\rangle$ at $\beta=0$ characterizes the average fractional population imbalance for polaritons along the cavity length when the supercurrent $I_{\mathrm{c}}=0-$ see Eq. (16). The curve AB determines the transition from the MQST regions in Fig.1a to the region 5 of the Josephson oscillations in Fig.1b. Then, with increasing the absolute value of $\beta$-parameter, the regions 5 and 4 are uni- 
fied in phase plane and form a new drop-like region where the system is self-trapped (is not shown in Fig.1). Such an evolution of the phase portrait in phase-plane is accompanied by sharp transition presented in Fig.3b near the value of $\beta \approx 1.2$. Therefore, the drop-like region is collapsed to stationary point $S_{2}$ in Fig.1c with further expanding of the $\beta$-parameter. The curve BC in Fig.3b describes a switching effect from the MQST state with locked phase to the running phase MQST states determined by the region 1 in Fig.1c.

The switching dynamics of polaritons depending on the $\beta$-parameter has an important feature in the framework of polariton condensation problem for atomic system. In particular, for initially non-equilibrium state of polaritons in the cavity with large momentum $k_{\|}$, the variation of $\Lambda$-parameter can be used in experiment to achieve a quasi-condensation with $k_{\|}=0$ for low-branch polaritons. In turn, the last parameter can be easily varied in the experiment by changing the total number $N_{\text {ex }}$ of polaritons in the cavity (we can change, for example, the intensity of laser field interacting with the atoms). Thus, observed dynamical regimes for both fractional population imbalance $\varsigma$ and relative phase $\theta$ allow, first, to estimate the fraction of condensed polaritons in the cavity taking into account the relevant expression (16) for the current $I_{\mathrm{c}}$ and, second, to manipulate with those coherence properties.

\section{Conclusion}

In the paper we developed a quantum theory of Josephson dynamics for two macroscopic polariton modes (belonging to upper and lower energy branches being coupled together due to Kerr-like nonlinearity of atomic medium). The phenomenon can be used to examine the macroscopic superfluid properties of condensed polariton state as well. The analogy with nonrigid pendulum behavior has been carried out to understand the different regimes of polariton interaction. We found out the dispersion relations and the characteristic frequencies for various regimes of collective excitations in the cavity including the several effects, i.e. the Josephson and Rabi oscillations, different kinds of the MQST states for fractional relative population imbalance $\varsigma$. Some of them can be represented as the new one, and have not been considered yet.

The realization of macroscopic tunneling and interbranch interaction for polariton modes is important to study in experiment the relative phase coherence in the presence of polariton condensation (and/or quasi-condensation).

Acknowledgements This work was supported by the Russian Foundation for Basic Research (projects Nos. 04-0217359, 05-02-16576) and the Federal Programs of the Ministry of Science and Education of Russian Federation. Au- thors are very grateful to Referees for the valuable comments. A.P.A. is grateful to Mikhail Glazov for fruitful discussions.

\section{References}

1. J. Keeling, F. M. Marchetti, M. H. Szymanska, P. B. Littlewood: Semicond. Sci. Technol. 22, R1 (2007).

2. T. Brandes: Phys. Rep. 408/5-6, 315 (2005).

3. L. Solymar, Superconductive Tunneling and Applications (Chapman and Hall, London 1972).

4. I. Zapata, F. Sols, A. J. Leggett: Phys. Rev. A 57, R28 (1998).

5. S. Raghavan, A. Smerzi, S. Fantoni, S. R. Shenoy: Phys. Rev. A 59, 620 (1999).

6. Y.-B.Zhang, H. J. W. Muller-Kirsten: Eur. Phys. J. D 17, 351 (2001).

7. F. Meier, W. Zwerger: Phys.Rev. A 64, 033610 (2001).

8. R. Gati, M. Albeiz, J. Folling, B. Hemmerling, M. K. Oberthaler: Applied Phys. B 82, 207 (2006).

9. A. Yu. Leksin, A. P. Alodjants, S. M. Arakelian: Optics and Spectr. 94, 768 (2003).

10. E. A. Ostrovskaya, Y. Kivshar, M. Lisak, B. Hall, F. Cattani, D. Anderson: Phys. Rev. A 61, 031601 (2000).

11. H. Deng, G. Weihs, C. Santori, J. Bloch, Y. Yamamoto: Science 298, 199 (2002).

12. Le Si Dang, D. Heger, R. Andre, F. Boeuf, R. Romestain: Phys. Rev. Lett. 81, 3920 (1998).

13. M. Richard, J. Kasprzak, R. Andre, R. Romestain, Le Si Dang, G. Malpuech, A. Kavokin: Phys. Rev. B 72, 201301(R) (2005).

14. J. Kasprzak, M. Richard, S. Kundermann, A. Baas, P. Jeambrun, J. M. J. Keeling, F. M. Marchetti, M. H. Szymanska, R. Andre, J. L. Staehli, V. Savona, P. B. Littlewood, B. Deveaud, Le Si Dang: Nature 443, 409 (2006).

15. G. Malpuech, D. Solnyshkov, H. Ouerdane, M. Glazov, I. Shelykh: Phys. Rev. Lett. 98, 206402 (2007).

16. V. A. Averchenko, A. P. Alodjants, S. M. Arakelian, S. N. Bagayev, E. A. Vinogradov, E. S. Egorov, A. I. Stolyarov, I. A. Chekhonin: Quantum Electronics 36, 532 (2006).

17. C. Ciuti: Phys. Rev. B 69, 245304 (2004).

18. A. P. Alodjants, S. M. Arakelian, S. N. Bagayev, I. A. Chekhonin, E. S. Egorov: J. of Rus. Laser Research 27, 400 (2006).

19. R. Miller, T. E. Northup, K. M. Birnbaum, A. Bocca, A. D. Boozer, H. J. Kimble: J.Phys.B: At. and Mol. Opt. Phys 38, S551 (2005).

20. V. M. Akulin, W. P. Schleich: Phys. Rev. A 46, 4110 (1992).

21. S. Jiang, S. Machida, Y. Takiguchi, Y. Yamamoto, H. Cao: Appl. Phys. Lett. 73, 3031 (1998).

22. A. Brunetti, M. Vladimirova, D. Scalbert, M. Nawrocki, A. V. Kavokin, I. A. Shelykh, J. Bloch: Phys. Rev. B 74, 241101R (2006).

23. F. Laussy, M. Glazov, A. Kavokin, D. Whittaker, G. Malpuech: Phys. Rev. B 73, 115343 (2006).

24. A. I. Tartakovskii, D. N. Krizhanovskii, D. A. Kurysh, V. D. Kulakovskii, M. S. Skolnick, J. S. Roberts: Phys. Rev. B 65, 081308(R) (2002).

25. F. Dalfovo, S. Giorgini, L. P. Pitaevskii, G. Stringari: Rev. Mod. Phys. 71, 463 (1999). 
26. E. L. Bolda, R. Y. Chiao, W. H. Zurek: Phys. Rev.Letts 86, 416 (2001).

27. M. J. Paz-Alonso, H. Michinel: Phys. Rev. Letts. 94, 093901 (2005).

28. M. Lax, Fluctuation and coherence phenomena in classical and quantum physics (Gordon and Breach, New York 1969).

29. G.-S. Paraoanu, S. Kohler, F.Sols, A. J. Leggett: J.Phys.B: At. and Mol. Opt. Phys 34, 4689 (2001). 